\title{
LOS APROVECHAMIENTOS TRADICIONALES DE LAS AGUAS DE TURBIAS EN LOS PIEDEMONTES DEL SURESTE DE LA PENIINSULA IBÉRICA: ESTADO ACTUAL EN TIERRAS ALICANTINAS ${ }^{1}$
}

\author{
María Hernández Hernández \\ Alfredo Morales Gil \\ Departamento de Análisis Geográfico Regional y Geografía Física \\ Universidad de Alicante \\ maria.hernandez@ua.es, alfredo.morales@ua.es
}

\section{RESUMEN}

Las sociedades del sureste peninsular adoptaron una particular ordenación del territorio (el aterrazamiento de laderas y los riegos de turbias) con la que se trataba de minimizar, entre otros, la escasez de precipitaciones y unos horizontes edáficos poco desarrollados. Un análisis diacrónico pone de manifiesto los factores que condicionaron la génesis de estos paisajes, pero también la desarticulación de estos sistemas de riego a partir de la segunda mitad del siglo veinte. El estudio de las causas de este abandono es fundamental para comprender su estado actual. Éste nos permitirá evidenciar, además de la pérdida paisajística y patrimonial que ello ha significado, el incremento de una serie de problemas ambientales, que ésta trataba de atenuar (laminación de las ondas de crecida, pérdida de suelos, etc.).

Palabras clave: Sureste peninsular, aterrazamientos, riegos de turbias, abandono, paisajes agrarios, recursos hídricos, patrimonio hidráulico.

Fecha de recepción: enero 2012.

Fecha de aceptación: noviembre 2012.

1 Este artículo se inserta en el Proyecto de Investigación «Modelos de desarrollo territorial y tipologías de consumo de agua potable en espacios turístico residenciales del litoral alicantino» (CSO2009-12772-C03-03) financiado por el Ministerio de Ciencia y Tecnología. 


\section{ABSTRACT}

Southeast Spanish societies adopted a particular land management (terraced hillsides and irrigation with muddy water) in order to minimize, among others factors, the rainfall scarcity and scanty developed soils. A diachronic analysis reveals factors that conditioned the genesis of these landscapes, but also the dismantling of these irrigation systems since the second half of the twentieth century. The study of this abandonment causes is essential to understand its current state. It will allow us to highlight the increase of environmental problems, that this management was blunt (rolling of flood waves, soil loss, etc.). In addition to the landscape degradation and heritage degradation that these processes have meant.

Key words: Southeast of Spain, terraced hillsides, irrigation with muddy water, set asside, agricultural landscapes, water resources, hydraulic heritage.

\section{LA ORDENACIÓN TRADICIONAL DE LOS PAISAJES AGRARIOS EN EL SURESTE PENIN- SULAR, UNA SISTEMATIZACIÓN BASADA EN LA CONSERVACIÓN DE LOS SUELOS Y EL APROVECHAMIENTO INTEGRAL DE LOS RECURSOS HÍDRICOS}

En el sureste peninsular, los rasgos climáticos (precipitaciones exiguas, con unos totales anuales en torno a $300 \mathrm{~mm}$, y espasmódicas, ya que la mayor parte del total anual puede concentrarse en unos cuantos acontecimientos lluviosos de la estación otoñal) combinados, en ocasiones, con las disposiciones del relieve (fuertes pendientes) y las litologías dominantes (calizas y margas), que se traducen en suelos con horizontes edáficos poco desarrollados y fácilmente deleznables, no ofrecían muchas posibilidades para que sobre ellos se diese un intenso proceso de ocupación humana, puesto que la indigencia pluviométrica y la pobreza edáfica representaba un factor limitante de primer orden. Sin embargo, desde la Prehistoria fue ocupado este territorio debido, sobre todo, a unas buenas condiciones térmicas invernales e, incluso, se convirtió en un espacio desde el que se irradiaron algunas corrientes culturales como fueron las técnicas de los pueblos argáricos.

Estos rasgos determinaron las directrices básicas que las comunidades campesinas tuvieron que adoptar para hacer viable un aprovechamiento racional del territorio que les permitiera su supervivencia. En una sociedad como la tradicional, donde la agricultura es la base económica, suelo y agua adquieren particular relevancia. Por ello, cuando el medio no oferta, en la medida suficiente esos elementos, intentar mantener uno y acrecentar el otro, es la máxima que ha guiado las actuaciones antrópicas en estas tierras semiáridas. Éstas se sintetizaban en una doble aptitud: de una parte, buscar los medios para aprovechar las lluvias, generalmente concentradas en unos cuantos acontecimientos de la estación otoñal y, de otra, controlar su capacidad erosiva sobre los suelos y adoptar las medidas necesarias para reducir los coeficientes de escorrentía que se generan en los diferentes lechos y, de este modo, minimizar la pérdida de horizontes edáficos superficiales y más meteorizados. El riego se convierte, por tanto, en una necesidad para asegurar unos rendimientos adecuados de los cultivos, pero también para poder aprovechar al máximo los escasos recursos hídricos disponibles. Donde la irrigación continua no es posible, se mejora la humedad del suelo, concentrando el agua de lluvia en unas cuantas parcelas seleccionadas. Proceso que se produce de forma natural en los fondos de los valles, llamados 
aquí cañadas, donde se produce la convergencia de las escorrentías laterales de las vertientes que las circundan.

La conservación de suelo y la acumulación de agua era una preocupación manifestada por agrónomos y escritores romanos como Columela o Plinio, con antecedentes empíricos en Mesopotamia, Egipto y el pueblo nabateo, conscientes de la trascendencia que el suelo y el agua tenía en sociedades de base agrícola y en un medio donde predominaban los horizontes edáficos poco desarrollados y unas precipitaciones escasas y concentradas en el tiempo. Consecuentemente, se puede afirmar que disponibilidades hídricas y ordenación del territorio en esta región natural han ido siempre íntimamente unidas. Es, por tanto, el agua y su control el elemento que prima en la organización del sureste peninsular.

En estos territorios, además de los factores físicos, concurren otros relacionados con sus pobladores, que resultan no menos decisivos. Entre los condicionamientos humanos, destaca la presión demográfica como una constante que implicaba una demanda creciente de la superficie cultivada, a costa de interfluvios o piedemontes, que, en ocasiones, presentan pendientes en torno a 20 ó 30 grados. En unas civilizaciones con escasa tecnología disponible, el incremento de la producción sólo se logra cultivando nuevas tierras. El proceso conocido como hambre de tierras alcanza cotas insospechadas en el siglo XVIII cuando el notable crecimiento poblacional, se traduce en una fiebre roturadora encaminada a la obtención de los medios necesarios para la subsistencia. Se ponen, de este modo, en cultivo áreas de montaña hasta altitudes que, en ocasiones, se sitúan próximas al umbral ecológico de los cultivos, cuando no lo superan.

\section{LA ORDENACIÓN DE LOS ESPACIOS SEMIÁRIDOS MEDITERRÁNEOS, UNA SISTEMATIZA- CIÓN BASADA EN LA IMPLEMENTACIÓN DE TÉCNICAS VARIAS}

El agricultor, para minimizar los rasgos citados, ideó una sistematización del territorio que es el resultado de una doble necesidad: disponer de tierras para el cultivo y suministrar a las mismas caudales, al menos en una cantidad que permita asegurar las cosechas, lo que no siempre resulta factible. Ello fue posible mediante la adopción de sistemas que permitían captar los volúmenes circulantes por las ramblas y barrancos coincidiendo con aguaceros copiosos; pero, también, otros orientados a la retención de la escasa humedad caída directamente sobre las parcelas o superficies aledañas a ellas a mayor cuota altimétrica. Para ello, se derivan los caudales circulantes con ocasión de aguaceros intensos, las denominadas aguas de turbias; llegándose de este modo a realizar lo que se conoce como inundación dirigida o cosecha de agua (Martínez de Azagra, 1996). Mediante la inducción y «recolección» de la escorrentía que discurre por laderas, vertientes y cauces, se lograba almacenar la máxima cantidad de agua que fuera posible en el suelo durante los meses relativamente húmedos (otoño, invierno y primavera). Posteriormente, mediante la realización de diversas labores del suelo, se reducía la evapotranspiración por capilaridad. De este modo, se conseguía conservar la mayor cantidad posible de humedad y durante el máximo tiempo posible en los perfiles profundos del suelo e incrementar las disponibilidades hídricas para los cultivos.

Laderas, vertientes, lomas, barrancos y cauces fueron organizados y ordenados de manera que, las escorrentías se pudieran conducir y derivar hacia las parcelas de cultivo situadas en cotas inferiores. Esta ordenación se llevó a cabo mediante la adopción de prácticas diversas: 
1. Escalonamiento de las parcelas en el sentido descendente de la pendiente. Ello fue posible mediante la construcción de caballones o muros de mampostería perpendiculares a ésta. Así, se rompía la escorrentía y se obligaba al agua caída sobre el terrazgo a acumularse en la parte superior. Sólo si la lluvia era muy abundante, se podía producir un desbordamiento lateral hacia la parcela inferior y, para que no se generasen efectos erosivos sobre el caballón, se disponía en el mismo de una abertura llamada sangrador (figura 1), que se colocaba en el centro o en los extremos. Así, los excedentes acuosos podían ir pasando de bancal en bancal, sin erosionar y reduciendo al mínimo la pérdida de suelo.

Figura 1

ESQUEMA DE ESCALONAMIENTO DE LAS PARCELAS MEDIANTE CABALLONES Y SANGRADORES (IZQUIERDA) Y EJEMPLO DE SANGRADOR (DERECHA)
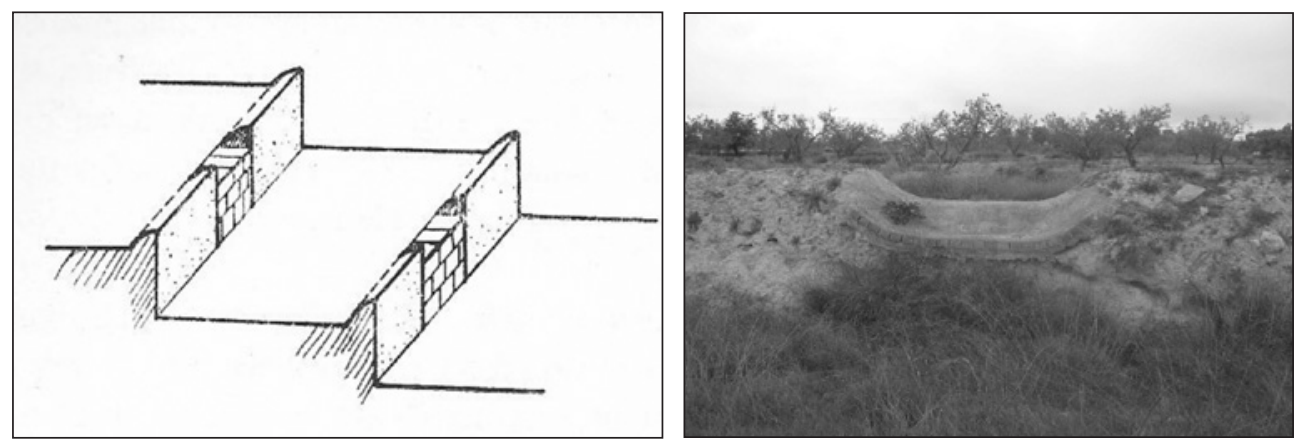

Fuente: Morales, 1969: 169 (gráfico de la izquierda) y Mondéjar, 2010 (imagen de la derecha).

2. Aprovechamiento de escorrentías difusas aguas arriba de las parcelas de cultivo. Las aguas circulantes por las laderas a mayor cuota altimétrica y que rodeaban a los terrazgos de cultivo se canalizaban hacia éstos. Se trataba de ordenar una escorrentía difusa con fines agrarios. De este modo, se incrementaba el volumen que estaba recibiendo ésta, mientras duraba el chubasco. Es lo que se conoce como riegos de vertiente. Ello fue posible mediante la realización de pequeñas actuaciones (caballones escalonados de disposición oblicua a la pendiente y confluentes en el punto más bajo de la ladera, de donde, arrancaba una boquera), que evitasen el desvío lateral fuera del área a cultivar, y limpiando de maleza la superficie inmediata a las mismas, a fin de favorecer la llegada de una lámina de agua difusa a la parte superior de la parcela.

3. Aprovechamiento in situ de los caudales circulantes por el lecho. Este procedimiento, denominado parat en tierras valencianas, consiste en la elevación de un muro normalmente de tierra compacta y mampostería de hasta 8 ó 10 metros de altura y dispuesto de manera perpendicular a la dirección de los flujos (figura 2). Con su construcción, se provocaba, por un lado, la interceptación de éstos y su conducción, mediante un sangrador, hacia uno de sus extremos de donde partía un canal que actuaba de punto de descarga del agua retenida, dirigiéndola a los campos cultivados situados a un nivel inferior y, por otro, se favorecía la deposición, aguas arriba, del material sólido que el agua transportaba, con lo que se incenti- 
vaba el desarrollo de horizontes edáficos. La deposición de sedimentos arenosos y arcillosos genera con el paso del tiempo la colmatación de la pequeña presa, con lo que se crean nuevos espacios agrícolas. Al mismo tiempo, en la parte inferior de la pequeña presa aterrada, se producía una convergencia de aguas subálveas inducidas por dichos sedimentos, lo que permitía disponer de unos pequeños caudales que se aprovechaban durante la estación seca e, incluso, se almacenaban en una pequeña alberca. A modo de ejemplo, cabe citar el parat de Catxuli, o los de la rambla de Puça o de la Zarza (Petrer), el de la Vall de Ebo o el parat de rec en Callosa d'en Sarria.

Figura 2

PRESA LEVANTADA EN EL LECHO DE LA RAMBLA DE PUÇA (PETRER, ALICANTE)

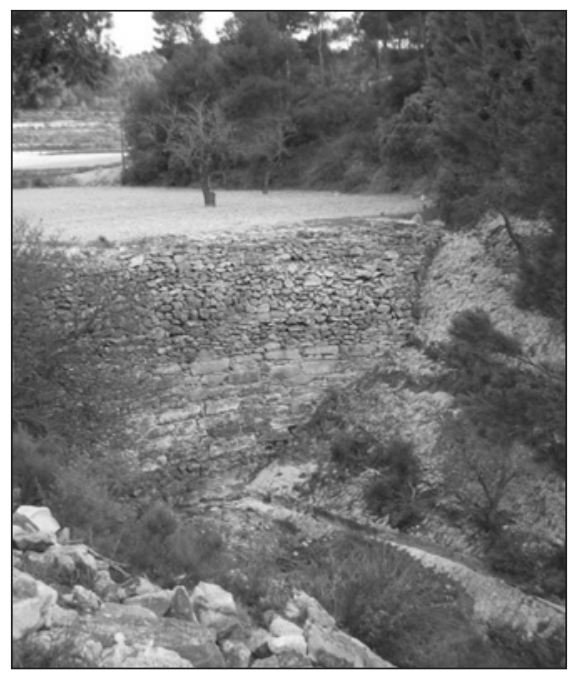

Fuente: Hernández, 2010.

4. Derivación de caudales espasmódicos circulantes por ramblas y barrancos. Cualquier cauce, por pequeño que fuese, era susceptible de una «corrección hidrológica», con el fin de aprovechar sus aguas y de laminarlas en los bancales agrícolas para beneficio de las plantas cultivadas. La trascendencia de estos aportes esporádicos era tal en un medio semiárido, que el agricultor debía utilizarlos al máximo, no pudiendo dejar que se perdieran en el cauce de las ramblas. El método consistía básicamente en la instalación de una presa en el lecho de estos cursos fluviales de flujos circulantes intermitentes (figura 3), generalmente de mampostería que provoca la interceptación de una parte de la arroyada, la cual es desviada hacia un canal lateral al que, propiamente se denomina boquera (Morales, 1969: 170). Su distribución, una vez extraídas del cauce fluvial, se realiza mediante una red cuya complejidad depende de la importancia de la superficie a regar (figura 4). En esencia, consta de una acequia principal de la que derivan los brazales encargados de dirigir las aguas hasta los bancales. Sistema de irrigación que puede considerarse casi perfecto pues, incluso, estaba prevista la devolución del caudal sobrante, el cual retornaba a la rambla o a otras acequias de riego y permitía su aprovechamiento aguas abajo. La importancia que 
estos caudales ocasionales tenían para estas sociedades de base agrícola configuró sistemas de cierta complejidad, ya que era frecuente la instalación de diversas presas a lo largo de un mismo barranco. En el lecho de la rambla de Vergeret, entre los términos municipales de Alicante, Busot y Xixona, por ejemplo, existieron un total de 17 sistemas de riego con boqueras (Mondéjar, 2010).

Figura 3

ESQUEMA DE LA INSTALACIÓN DE UNA PRESA EN EL LECHO DE LA RAMBLA Y EJEMPLO DE BOQUERA EN EL CAMPO DE ALICANTE
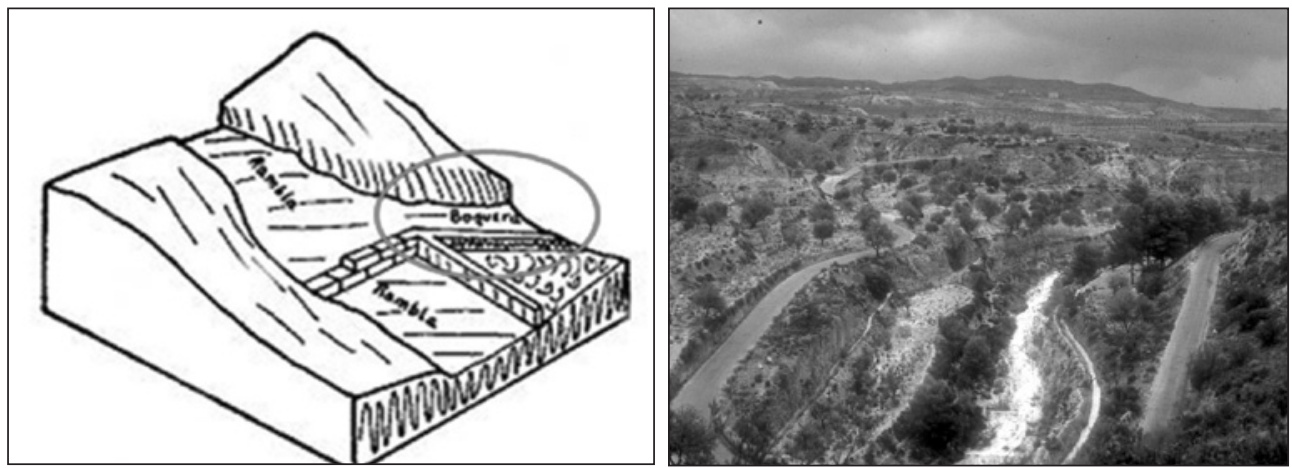

Fuente: Morales, 1969: 172 (gráfico de la izquierda) y Morales, 2010 (imagen de la derecha).

Figura 4

RED DE DISTRIBUCIÓN DE CAUDALES A PARTIR DE UNA BOQUERA EN LA RAMBLA DEL PUERTO DE GARRUCHAL (MURCIA), DESTRUIDO EN LOS ÚLTIMOS AÑOS COMO CONSECUENCIA DE LA EXPANSIÓN RESIDENCIAL SOBRE EL ANTIGUO USO AGRÍCOLA
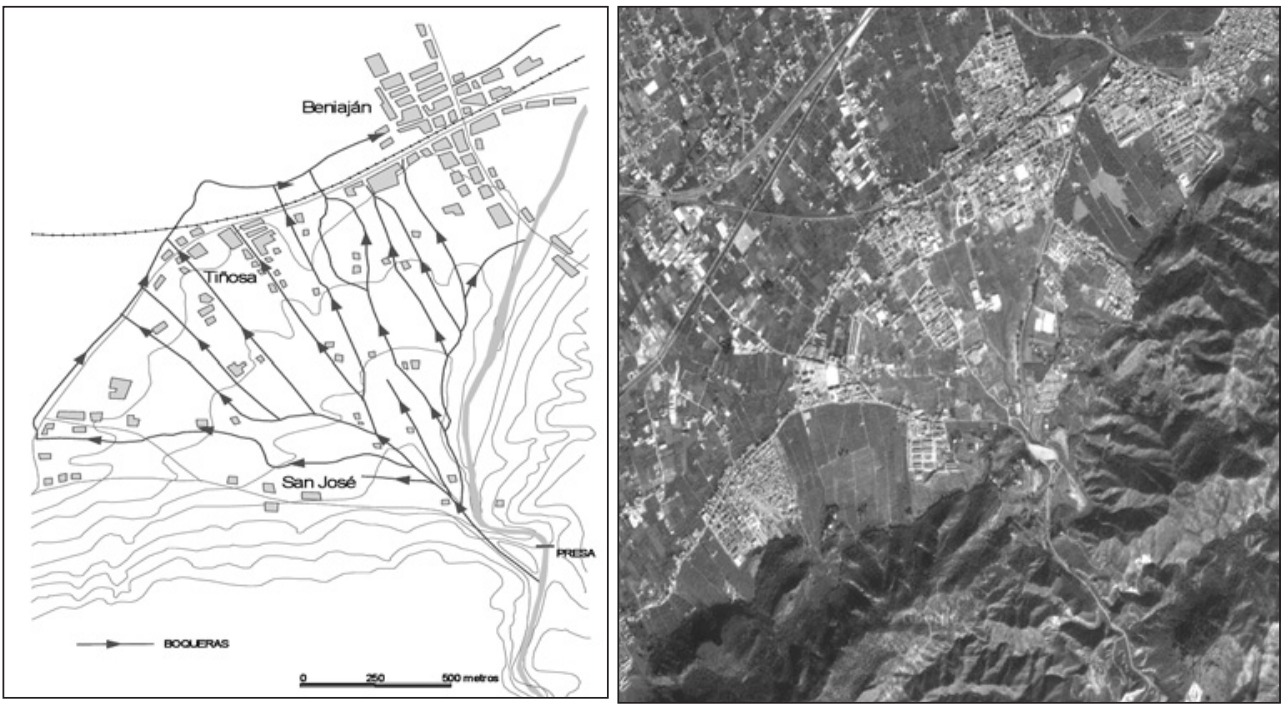

Fuente: Morales, 1969: 175 (gráfico de la izquierda) y Googlemaps, 2007 (imagen de la derecha). 
5. Sistemas mixtos: boqueras y aterrazamiento de laderas. Los sistemas de riegos de boquera se asocian, en numerosas ocasiones con el aterrazamiento de las laderas (figura 5). Con su combinación se consiguen dos beneficios, aumentar los recursos hídricos y «controlar» las pendientes. El empleo del aterrazamiento es, quizás, la expresión más directa de la necesidad de conservar el suelo y la humedad en áreas de montaña. Siendo considerado como uno de los sistemas más antiguos y eficaces para disminuir la erosión al modificarse el régimen hidrológico de la ladera mediante la construcción de parcelas escalonadas con superficies horizontales o subhorizontales, que provocan una modificación de las pendientes y del flujo circulante por las mismas, ya que dada su ligera inclinación hacia el interior de la parcela se favorece la percolación. Con este sistema, el suelo es capaz de retener una parte importante de los volúmenes aportados directamente por las precipitaciones o por la escorrentía, al tiempo que se evita la pérdida de suelos generada por una circulación libre de las aguas. Las terrazas remontan así las pendientes como graderías que ascienden en ocasiones hasta la misma cumbre y, como norma general, hasta el límite ecológico de los cultivos.

Figura 5

ORDENACIÓN DEL PARAJE DE LA PRESA DE LA REVUELTA (BARRANCO BLANCO, ALICANTE), DONDE SE COMBINA EL RIEGO DE BOQUERAS Y ELABANCALAMIENTO DE LAS LADERAS (DERECHA) Y LADERA ATERRAZADA
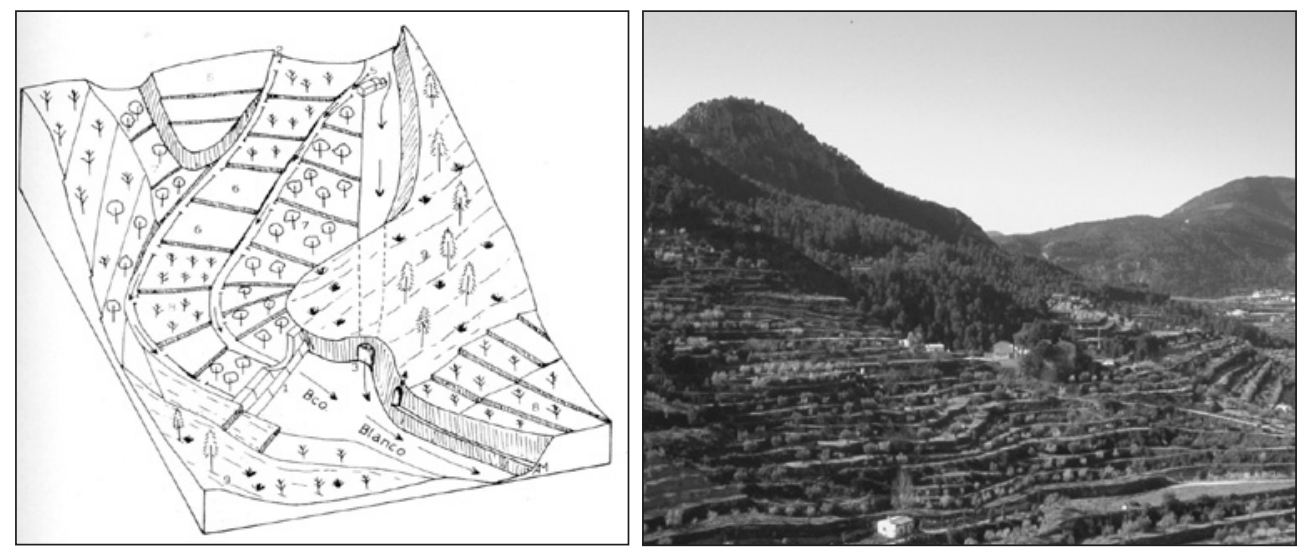

Fuente: Morales y Box, 1986: 19 (gráfico de la izquierda) y Hernández, 2009 (imagen de la derecha).

6. Galerías drenantes. En la búsqueda de recursos hídricos para permitir el asentamiento de las poblaciones rurales, se buscó complementar los volúmenes de agua permanente mediante la construcción de galerías drenantes. Son túneles con pendiente suave (un 0,5 por mil) que conducen el agua acumulada sobre los sedimentos cuaternarios hacia la superficie del suelo por simple gravedad (Hermosilla, 2006). Éstas, construidas en horizontal con pozos verticales asociados, reciben diferentes denominaciones qanats, kettara, foggara, galería con lumbreras, etc. (Gómez, 2006:18). Muchas de ellas construidas en los siglos XVIII y XIX vinculadas a la expansión de las tierras cultivadas; aunque, algunas cabe retrotraerlas a la dominación musulmana, como la existente en el antiguo poblado morisco de la Puça, de la que a principios del XVII quedaba el topó- 
nimo (Tros de l'Alcavó) y un croquis en que se le denomina la Mina Cega, topónimo que aún perdura (Bernabe i Mestre, 1989). Estos sistemas, que se excavaban en los glacis y en los lechos fluviales de ramblas y barranco, aportaban en ocasiones volúmenes de agua entre 1 y 10 l/seg. y permitieron el abastecimiento de unos conjuntos poblaciones del entorno de los 20 a 100 habitantes.

7. Construcciones para acumular aguas pluviales. La escorrentía de laderas montañosas, e incluso de barrancos, se captaba y conducía mediante pequeñas boqueras para acumular el agua circulante en aljibes, balsas y estanques (Box, 1995). Con los primeros, se pretendía almacenar el agua necesaria para la población que vivía en estos territorios durante todo el año. Con las segundas, se atendía temporalmente a las necesidades hídricas de los ganados y animales de tracción. Los terceros se utilizaban para los mismos fines de las segundas y, además, para irrigar pequeños huertos, que abastecían temporalmente de hortalizas a los habitantes de estos poblamientos rurales dispersos. Estos sistemas de captación conllevaban la realización de tareas varias en la cuenca de la que se derivarían los caudales. Previamente, se reducía la vegetación existente para reducir los consumos asociados a éstas. Desde aquí las aguas eran conducidas a través de una boquera a un decantador. Dada la elevada turbidez de los caudales circulantes era necesaria su construcción para que en ellos se depositasen los lodos, con lo que se incrementaba el volumen de agua útil almacenada. Por último, mediante un pequeño canal entran en el aljibe o en la balsa (figura 6).

Figura 6

ESQUEMA DE LA ORDENACIÓN DE UNA BOQUERA PARA LLENAR ALJIBES. EJEMPLO DE UN ALJIBE EN EL PARAJE DE RINCÓN DEL MORO (VILLENA)
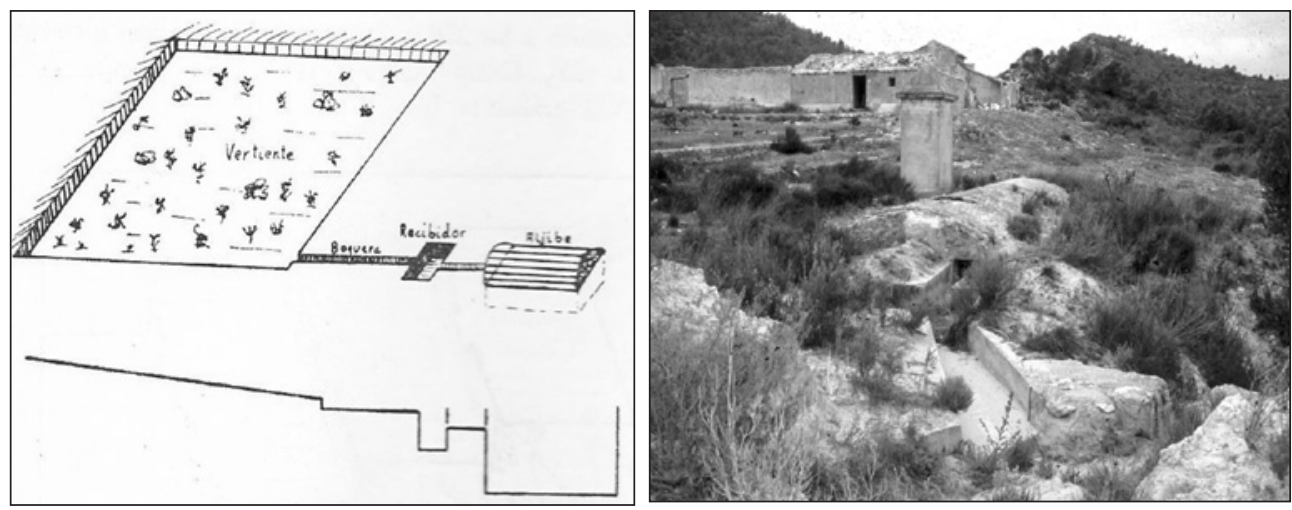

Fuente: Morales, 1969: 178 (gráfico de la izquierda) y Hernández, 2009 (imagen de la derecha).

\section{LOS PAISAJES DEL «SECANO MEJORADO»: DISTRIBUCIÓN ESPACIAL Y REFERENCIAS HISTÓRICAS}

Este conjunto de actuaciones permitió la ordenación de grandes superficies, que en el caso del sureste peninsular implicó la casi totalidad del territorio donde se practicaba el cultivo pluvial secano. El sistema de riegos de turbias creó, lo que algunos autores denominaron como «secano mejorado» (López Gómez, 1951), es decir, se configura como un tipo 
de aprovechamiento intermedio entre el secano y el regadío, ya que esos mayores aportes hídricos permitían reducir la aleatoriedad del primero, pero en ningún caso consentían el desarrollo de cultivos de regadío en sentido estricto. Consintió un dificultoso desarrollo de plantaciones de olivos, almendros, algarrobos e incluso frutales, $\mathrm{y}$, mediante la práctica del barbecho, el cultivo de cereales de pocas exigencias en humedad. Esta ordenación del territorio se completaba con la adopción de diversas técnicas orientadas a mejorar las condiciones naturales que las exiguas lluvias aportaban. El sistema de cultivo asociado (vuelo -arbolado- y suelo -cereales-) era otra técnica adoptada por los agricultores para minimizar el impacto de una reducción significativa de las precipitaciones: la pérdida de la cosecha de cereales podía compensarse con la obtenida de los cultivos arbóreos y en años secos o muy secos, ésta última era la única obtenida (figura 7). El predominio de los cultivos arbóreos (olivos, algarrobos, higueras y almendros, entre otros frutales) es otra característica de estos paisajes. El desarrollo de unos sistemas radiculares profundos y de gran extensión, marcos de plantación amplios (de 8-10 metros en los olivos, lo que equivale a unos 110 pies por hectárea y de hasta 15-20 metros en los algarrobos, unos 30-40 pies por hectárea), permitía la supervivencia del arbolado y asegurar, al menos una parte de la cosecha en periodos de sequía. Esos mismos marcos amplios de plantación facilitaba el doble sistema de cultivo (vuelo y suelo).

Figura 7

PARTIDA DE LA CONDOMINA (HUERTA DE ALICANTE, 1944). LOS APROVECHAMIENTOS DOMINANTES, LAASEMEJAN MÁS A UN SECANO QUE A UN REGADÍO TRADICIONAL.

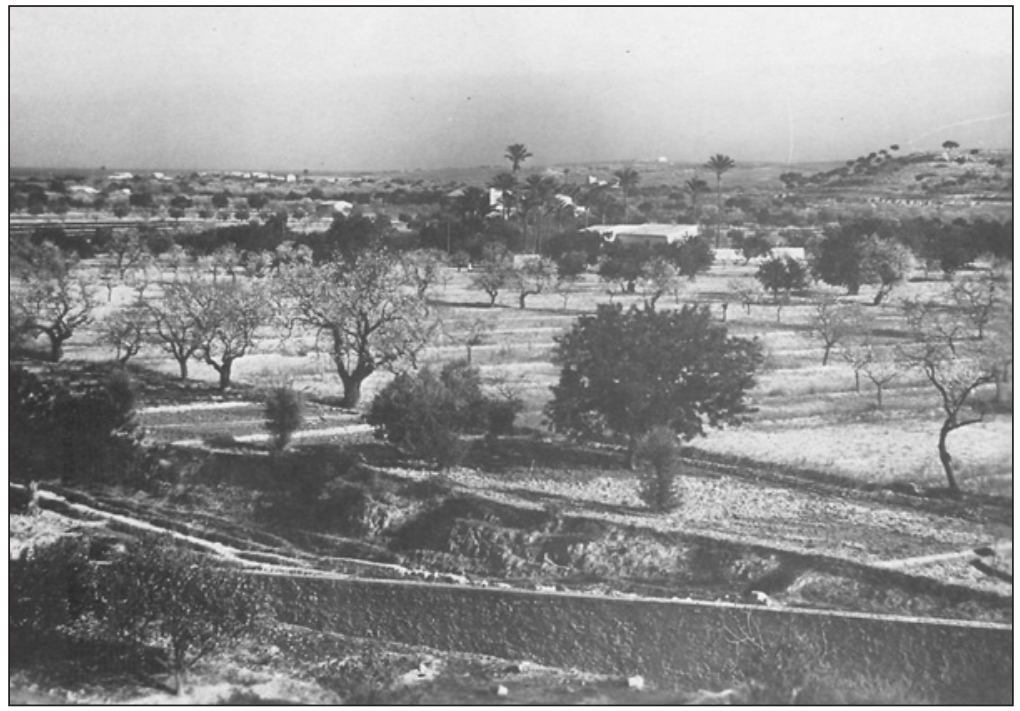

Fuente: Román del Cerro, 1984: 148.

La lucha contra la aridez y la rápida escorrentía, ya aportando agua a los cultivos en épocas de sequía mediante rudimentarios sistemas de regadío eventual, ya parcelando en terrazas las vertientes para corregir los procesos erosivos, podría retrotraerse a la coloni- 
zación agraria del Neolítico como han afirmado diversos arqueólogos (Chapman, 1981). Su existencia queda totalmente corroborada en época romana, como se desprende de la impronta que dejaron en el parcelario las centuriaciones romanas en Jumilla, Yecla, Sax, Elche o los restos de presas como la de Román en la rambla del Moro, entre los términos de Abarán y Jumilla. Un aprovechamiento que fue extendido y desarrollado durante la ocupación árabe y así se recoge en el Libro del Repartimiento de la Huerta y Campo de Murcia ( siglo XIII), en el que se alude a áreas de cultivo que se benefician de aguas eventuales a la que se designa como riegos de alfait, término que significa literalmente crecida (Torres, 1960: 279). La posterior expulsión de los moriscos supuso el abandono de vastas superficies generalmente marginales, que permanecieron durante largo tiempo incultas por falta de mano de obra. Proceso que se invertirá durante los siglos XVIII y XIX. Centurias en las que debido al incremento demográfico, se inició un proceso roturador que se vio favorecido por la desamortización de bienes eclesiásticos y de propios y que se traducirá en la puesta en cultivo de amplias áreas de glacis que hasta ese momento sólo se aprovechaban como espartizales o para la explotación de leñas.

La trascendencia de estos aportes esporádicos en unos territorios semiáridos queda patente en diversos medios (procedimientos o normativas, tasas o impuestos pagados por las tierras, toponimia, mapas o planos, etc.). En numerosos documentos históricos, se hace referencia a estos sistemas de derivación: escrituras públicas que recogen a los propietarios de estas infraestructuras, otros donde se mencionan las diversas técnicas constructivas o las tareas de mantenimiento. De igual forma, se establecieron normas para su equitativo reparto, así como disposiciones que preservaban los derechos de los dueños de los predios inferiores. Un primer texto que hace referencia a la manera en que han de utilizarse las aguas de avenida es el Fuero Juzgo o Libro de los Jueces (siglo VII), el cual indica que: «nengun omne debe encerrar el rio por toller la pro a todos los otros, é facerla suya; mas puede facer seto fasta medio del rio, alli o es el agua mas fuerte, é que la otra meatad finque libre para la pro de los omnes» (Morales, Box y Marco, 1989). Igualmente, en El Código de las Siete Partidas (1256-1263) figura el modo de aprovechar las aguas de escorrentía difusa: «El dueño de la heredad superior puede retener en ellas las aguas de lluvias y otras semejantes para darles el destino que más le acomode, aunque el dueño de la heredad inferior las hubiere aprovechado siempre y salvo que tuviese título constitutivo que le diese el derecho a tomarlas al salir del predio más alto». También otros tipos de normativas reguladoras locales como, por ejemplo, las Ordenanzas del Campo y las Huerta de Murcia, aprobadas por Carlos II (1695), las Ordenanzas y Privilegios de la Muy Noble y Leal Ciudad de Lorca (1713) o el Reglamento para el aprovechamiento de las aguas de Riego de la Huerta de Alicante (1849) hacían mención a la utilidad del aprovechamiento de estos caudales (Mondéjar, 2010) así como a las normas que regulaban su uso. Las primeras disposiciones legales que se dictan en España en materia de aguas, a finales del siglo XIX, también tienen muy en cuenta la utilización de aguas de avenida. Así el Real Decreto de abril de 1860, previo a la primera Ley de Aguas de 2 de agosto de 1866, indica: «El aprovechamiento de las aguas torrenciales debe estimularse pues proporciona á las tierras humedad y abonos, evitando que se pierdan en los mares las sales y el mantillo, que son el alma de la vegetación; y lejos de perjudicar a los dueños de prédios inferiores, les favorece, evitando ó precaviendo las inundaciones y la destrucción de los 
muros de defensa. Pero para esto hay que cuidar con especial esmero de que con el uso de esta clase de aguas no lleguen á lastimarse los intereses legítimos de los aprovechamientos permanentes: las boqueras deben abrirse sobre el nivel ordinario de las aguas, y habrán de adoptarse otras varias precauciones» (Morales, Box y Marco, 1989). De igual forma, la Ley de Aguas de 1879, sucesora de la norma anteriores, regula el aprovechamiento, tanto de las aguas pluviales como las de escorrentías discontinuas. Permite, entre otras cosas, la construcción de aljibes en terrenos públicos, así como de «ribazos», «malecones»o «paredes» que, sin impedir el curso total de las aguas, sirvan para su laminación y aprovechamiento eventual.

Asimismo, la menor dependencia con respecto al secano le otorgaban un estatus intermedio entre las tierras irrigadas y las de secano en sentido estricto, lo que queda plasmado en las escrituras públicas al pagar una mayor contribución que el resto de las propiedades no regadas y apareciendo con esta distinción en los amillaramientos (Llobet, 1958). En el correspondiente a 1900 de Petrer, el 7,7\% de la superficie cultivada se recoge bajo el epígrafe de regadío esporádico. Su relevancia se acrecienta si aumentamos la escala: en la partida de la Almafrá, por ejemplo, de un total de 18 hectáreas, 17 corresponden a espacios beneficiados por las turbias (Payá, 1990). Referencias similares se encuentran en numerosos municipios adscritos a la región climática del sureste peninsular.

$\mathrm{Su}$ trascendencia se manifiesta, también, en el hecho de que el término de boquera o aquellos que se relacionan con este sistema de riegos eventuales (azud, derramador, partidor, etc.) son frecuentes en la toponimia de esta región climática. Referencias a la boquera de la Romana o Monóvar, la Casa de la Boquera de Bocairente, de Villena o Salinas, a la partida de les boqueres de San Vicente del Raspeig o la del boquerón (Sax), etc. son ilustrativas. Del mismo modo, aparecen recogidos dichos términos en representaciones cartográficas que reproducen propiedades rústicas (figura 8). La correspondiente a la finca del Azaraque, propiedad del Marqués de los Vélez, en el municipio de Alhama de Murcia, es representativa. Datada en 1760, la leyenda en la que se especifican los límites de la explotación hace referencia a términos como «boquera de aguas turbias», «azud del repartimiento de las aguas turbias» y «balsas». El análisis de este plano evidencia la localización de estas infraestructuras en el lecho de las ramblas, su trazado hasta las tierras que beneficia (olivar), algunas de reciente roturación, y la acumulación de excedentes en balsas. Su relevancia se prolonga en el tiempo. El análisis y fotointerpretación de la fotografía aérea correspondiente al vuelo americano (1956) permite poner de manifiesto la importancia que estos sistemas de aprovechamiento tuvieron en la región climática del sureste peninsular hasta aproximadamente los años cincuenta del siglo XX. Los riegos de turbias alcanzaron una amplia difusión en el Bajo Segura, en el Campo de Alicante, en el Alto y Bajo Guadalentín, en el Campo de Cartagena o en el valle del Almanzora. Representativos son los que se localizan en los glacis del Campo de la Matanza (Albatera) y en Granja de Rocamora, cuyos canales de derivación se estructuraban a partid del azud de Benferri, sobre la rambla del mismo nombre. En el Campo de Alicante, en los términos municipales de Agost y San Vicente cubrían amplias extensiones en los márgenes de la rambla de la Zarza, Alabastre, Pepior o barranco Blanco (Mondéjar, 2010). 


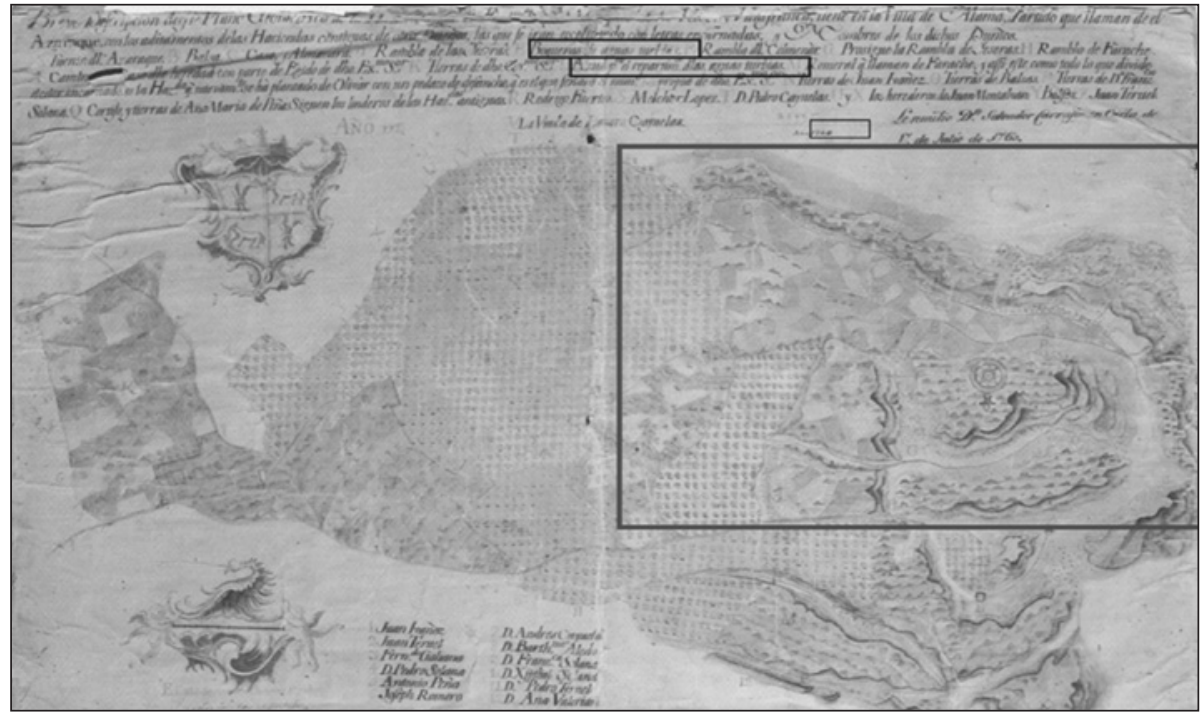

Fuente: Biblioteca Nacional.

La particularidad de estos sistemas de regadío, basados en el aprovechamiento de los caudales coincidiendo con episodios de lluvias o lo que se ha denominado como «regando cuando llueve» resulta sorprendente para los foráneos. Siendo recogido en numerosas obras de viajeros y naturalistas. Significativo resulta el párrafo del ilustrado Cavanilles quien a fines del siglo XVIII, escribía con relación a la zona de Agost lo siguiente: «quien ignore ser suma la escasez de agua en aquella parte del reyno, y que à veces un solo riego basta para asegurar y aumentar las cosechas, extrañara ver salir los labradores hácia sus haciendas cuando empieza á tronar, ó amenaza alguna tempestad: los truenos, que en otras parte del reyno sirven de señal para retirarse á sus habitantes lo son aquí para desampararlas y salir en busca de las aguas y deseado riego: se fecundan entónces los olivos, higueras, almendros, viñas y algarrobos: y el suelo entera se mejora con el cieno que traen las aguas» (Cavanilles, 1797: 524). Referencias al valor, significado y trascendencia del agua en estas tierras aparecen también de manera frecuente en las obras de Beramendi y Freire, que recorrió las tierras valencianas a finales del siglo XVIII (Soler, 1994), o en la de Richard Ford, quien afirmaba: «el agua aquí, de la misma manera que en oriente, es la sangre misma de la tierra y sinónimo de fertilidad y riqueza, su reparto se convierte en constante motivo de solicitud y diferencias» (Ford, 1982: 11).

\section{LA DESORGANIZACIÓN DE LA ORDENACIÓN TRADICIONAL DEL PARCELARIO EN EL SURESTE PENINSULAR, PÉRDIDA PATRIMONIAL Y PAISAJÍSTICA Y ACENTUACIÓN DE LOS RIESGOS NATURALES}

Una utilización del medio basada en un conocimiento profundo del espacio y su dinámica natural, fue lo que permitió adaptarse a las condiciones imperantes en el sureste español y, con 
ello, aprovechar al máximo cuanto del medio era posible, contribuyendo, a la vez, a controlar las arroyadas, pues la ruptura de pendientes por el abancalamiento, al tiempo que la desviación de caudales por las boqueras, implicaba una considerable reducción de los coeficientes de escorrentía y una enmienda orgánica y natural del suelo. Se conseguía con ello laminar y mermar los volúmenes de las crecidas y, en definitiva, una aminoración de los destructivos efectos de las avenidas fluviales en los tramos bajos. Igualmente, se creaban suelos agrícolas con la retención de légamos e incrementando la infiltración en los suelos, la recarga de acuíferos.

El paisaje resultante es, por tanto, y, ante todo, el fruto de costosísimos esfuerzos del agricultor que con su trabajo y un rudimentario instrumental, ha modelado laderas y cauces hasta límites insospechados, «el campo es aquí, sobre todo, arquitectura» (Deffontaines, 1972). Una trasformación, en definitiva, que implica no sólo un trabajo penoso, sino lo que es más de encarecer, interminable puesto que como afirma Braudel (1975) «un sólo instante de reposo y la montaña recobra su salvajismo primitivo y vuelta a comenzar». Esta afirmación evidencia que la creación de estos paisajes, resultado de una dilatada interacción entre la sociedad y el medio, obliga a unas tareas de mantenimiento constantes y su reducción o el abandono de las prácticas agrícolas producen notables repercusiones desde el punto de vista paisajístico y ambiental. La sociedad no sólo ha jugado un papel fundamental en la creación de estos paisajes, sino también en su conservación.

El abandono de estas áreas productivas marginales es consecuencia de varios factores. El primero, la baja rentabilidad de los cultivos de secano que no pudieron resistir la competencia salarial surgida en la costa, donde se instalan actividades secundarias y terciarias a mediados del siglo veinte, así como áreas de cultivo de regadío que producen frutos extratempranos de elevada cotización en los mercados nacionales y extranjeros; proceso que se traduce en una significativa reducción de los activos agrarios a los que hay que unir un importante éxodo rural. El segundo, va a ser la posibilidad de disponer de mayores volúmenes de agua, bien mediante la regulación de las escasas disponibilidades autóctonas, una mejor administración y reparto de los mismos, o la obtención de aportes subterráneos gracias a la utilización de bombas de gran capacidad e incluso procedentes de otras cuencas. La dificultad para mecanizar los parcelarios aterrazados contribuirá, asimismo, al abandono de aquellos terrazgos de menores dimensiones.

Figura 9

PROCESOS DE DESARTICULACIÓN E INTENSIFICACIÓN DE LOS PROCESOS EROSIVOS EN LA CUENCA DEL BARRANCO BLANCO (ALICANTE)
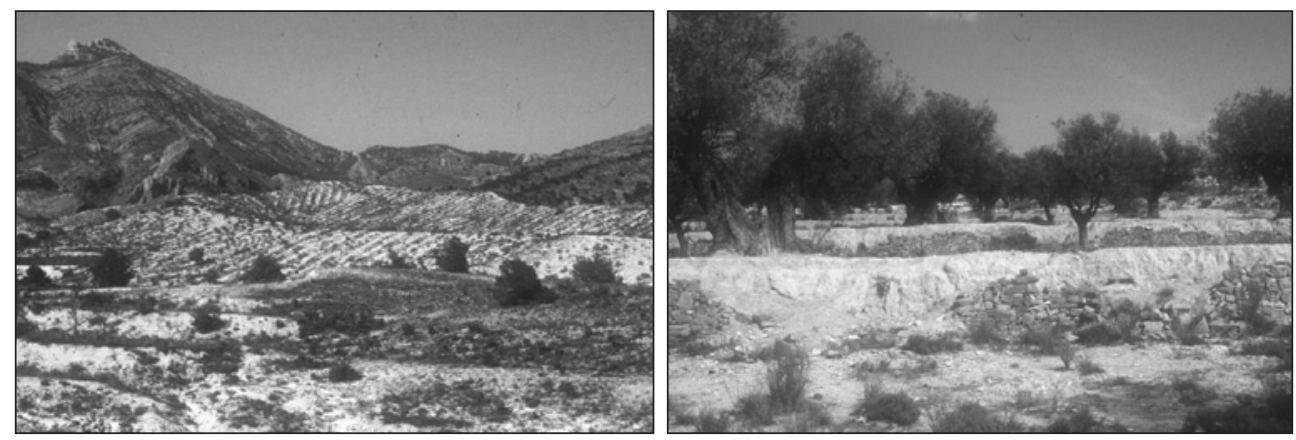

Fuente: Hernández, 2010. 
El abandono de estos terrazgos agrícolas como la omisión de determinadas tareas conlleva la progresiva destrucción de toda la sistematización. Éste no sólo se va a traducir en la desarticulación de estos paisajes, unas veces por abandono y otras por la implantación de sistemas de cultivo que no contaban con la incidencia de los fuertes aguaceros sobre los nuevos parcelarios, sino, también, la pérdida de las ventajas que esta ordenación generaba y, consiguientemente, la acentuación de aquellos procesos que minizaban. En primer lugar, el abandono de tareas (la no reparación de los caballones, muretes y presas o la limpieza periódica de las boqueras, entre otras) ha favorecido la reactivación de los procesos erosivos (figura 9). Hecho que se ve incrementando por la ausencia de vegetación y, sobre todo, porque las aguas de arroyada van a afectar a suelos removidos por la actividad agrícola anterior y que, consecuentemente, carecen de consistencia. Los mecanismos causantes de estos fenómenos se relacionan directamente con las circulaciones hídricas, sobre todo de aquéllas que lo hacen superficialmente en materiales de relleno cuaternario y en margas. Son frecuentes y expresivos enormes golpes de cuchara (Marco y Vera, 1988) en los bancales, en especial cuando estos presentan muretes y dominan los materiales relativamente heterogéneos; mientras que cuando predominan los finos, la forma más frecuente es la cárcava originada a partir de un caballón ya sin protección, progresando hacia el interior de la parcela a partir del salto.

La falta de las prácticas de mantenimiento ha supuesto, asimismo, una pérdida de la capacidad de laminación de las aguas de escorrentía, que era ejercida anteriormente por los aterrazamientos y las boqueras. De manera que, ahora cuando se producen chubascos de fuerte intensidad horaria, la arroyada superficial se ve acelerada en relación a su funcionamiento en los siglos anteriores, provocando un aumento considerable de los caudales circulantes y, lógicamente, potenciando la capacidad erosiva de estas ramblas. Resultado de todo lo indicado anteriormente es que las corrientes generadas por estos aguaceros adquieren una elevada capacidad de carga. Las consecuencias de esta situación se han dejado sentir no sólo allí donde estos fenómenos se han desencadenado, sino, como es lógico también en las partes más bajas de las cuencas vertientes. Ilustrativo resulta el anegamiento de áreas urbanizadas y áreas de equipamientos y servicios en las proximidades de la acequia del Gualeró, que distribuía las aguas de turbias derivadas a partir del azud de San Joan (Alicante).

Figura 10

ABANDONO Y DEGRADACIÓN SON LOS RASGOS QUE CARACTERIZANA LAS INFRAESTRUCTURAS ASOCIADAS A ESTOS PAISAJES DE TURBIAS: ESTADO DEL AZUD DE MUTXAMEL (IZQUIERDA) Y DE LA BOQUERA DE LA CASA DE BERENGUER (RAMBLA DE VERGERET) (DERECHA)
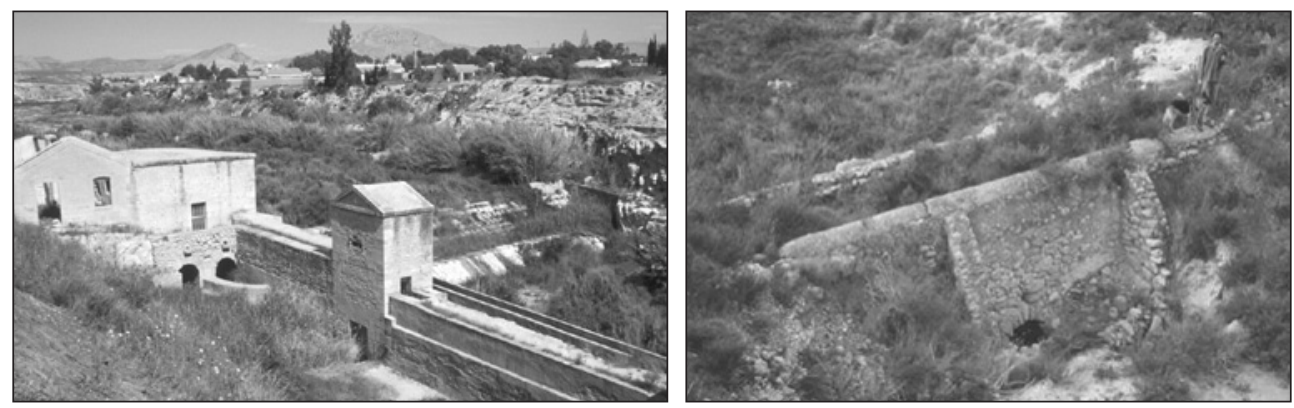

Fuente: Hernandez, 2010 y Mondejar, 2010 (figura de la izquierda y derecha, respectivamente). 
A los procesos de degradación ambiental e incremento de los riesgos naturales, se une la pérdida paisajística y patrimonial (figura 10). La degradación de los paisajes aterrazados y de los riegos de turbias conlleva una notable pérdida patrimonial, de identidad de un territorio ya que nos encontramos ante paisajes que muestran las relaciones de una sociedad con el medio en el que se asienta. Trascendental resulta también reflexionar sobre la intensidad del proceso: unos sistemas que tardaron siglos en configurarse, prácticamente han desapareciendo en cincuenta años. Pero, no sólo es llamativa la destrucción física de muchas de estas obras, lo es más el desconocimiento que de estos riegos tiene la mayor parte de la sociedad actual del sureste peninsular, que ha olvidado como funcionaban estos sistemas de turbias hasta la generación de sus abuelos. Ahora, se desprecian e, incluso, allí donde perduran, a pesar del abandono, se consideran molestas para los nuevos sistemas productivos, tanto agrarios de regadío como en áreas urbanizadas, por lo que en ocasiones asistimos asombrados a la destrucción de presas milenarias como la de Román en la Rambla del Moro o la bicentenaria del puerto de la Cadena en las proximidades del complejo sanitario de la Virgen de la Arrixaca, ambas en la provincia de Murcia.

\section{REFLEXIONES FINALES}

La ordenación de los paisajes en el sureste peninsular, basada en un conocimiento profundo del espacio y su dinámica natural por parte del hombre, fue lo que permitió adaptarse a las condiciones imperantes en estas tierras y, con ello, aprovechar al máximo cuanto del medio era posible, ejerciendo, al mismo tiempo prácticas de regulación de las ramblas, laminando y controlando sus crecidas, creando suelos agrícolas con la retención de legamos e incrementando la infiltración en los suelos y, en definitiva, la recarga de acuíferos. Todo un conjunto de ventajas que la sociedad actual parece haber olvidado al ordenar el espacio agrario atendiendo a principios de rentabilidad económica rápida. $\mathrm{Su}$ abandono, consecuencia de las transformaciones culturales, sociales, económicas, tecnológicas, que vienen registrándose durante el último medio siglo, ha superado a la capacidad de aprendizaje en el manejo mediante prácticas sostenibles del territorio de las poblaciones locales. Este proceso se ha traducido en una importante pérdida de saberes para gestionar un territorio.

Paralelamente, a estos procesos de pérdida de saberes relacionados con la gestión del territorio y la intensificación de las actividades agrarias y la difusión de los usos urbanoresidenciales en amplios territorios que poseían altos grados de naturalidad y/o ruralidad, que caracterizan a los países desarrollados en la segunda mitad del siglo XX, en los últimos treinta años se ha producido un «redescubrimiento» de los paisajes culturales. En este proceso desempeñan un papel fundamental los valores que estas sociedades les asignan, a saber, económico, ambiental y cultural. El primero, se vincula al hecho de que además de producir materias primas, ofertan servicios relacionados con la difusión de las actividades turísticoresidenciales. El segundo, relacionado con la creciente preocupación por su degradación del paisaje, al ser considerado como un recurso natural más; por lo que es contemplado, con mayor frecuencia, por amplias capas sociales como un bien escaso, difícilmente renovable y fácilmente degradable. El tercero, relacionado con elementos patrimoniales, culturales y de identidad, al ser reflejo de las relaciones entre una sociedad y su entorno. 
Diferentes colectivos (conservacionistas, técnicos medioambientales, agentes de desarrollo local de las administraciones, entre otros), se han planteado en las últimas dos décadas hasta que punto sería factible la restauración y el mantenimiento de los antiguos sistemas de aterrazamientos y riegos por boqueras, desde el punto de vista de las ventajas que aportan al medio cultural respetuoso con el físico (conservación de suelo, recarga de los acuíferos, laminación de las ondas de crecida, etc.), pero también desde una óptica económica (diversificación de las actividades económicas vinculadas a la conservación de estos paisajes) y cultural-patrimonial, muchas veces minusvalorado. En este sentido, lo realmente abrumador de los procesos de desorganización de estos paisajes, es el abandono que se genera, pues, un paisaje que ha tardado siglos en configurarse, que es el resultado del esfuerzo y sacrificio de muchas generaciones, se está destruyendo con tal rapidez que, si no se adoptan las medidas oportunas con prontitud, se perderá irremediablemente.

Las iniciativas orientadas a su conservación se han desarrollado al amparo de las políticas agrarias, particularmente a partir de los Programas de Desarrollo Rural 2000-2007 (programa LEADER, iniciativa LEADER y programa Cultura 2000) y los programas orientados a la mejora del medio ambiente, adoptados a mediados de los noventa (programa LIFE, por ejemplo). Así, la lucha contra la erosión es un tema de vital importancia en estas tierras subáridas y, en la mayoría de las ocasiones, se aborda exclusivamente mediante repoblaciones forestales. Las antiguas terrazas podrían conservarse y ser utilizadas para la replantación de especies arbóreas propias del clima mediterráneo. También son beneficiosos desde el punto de vista de la circulación de los flujos hídricos pues ésta quedaba en gran parte, contrarrestada y con ello se facilitaba la percolación en profundidad de las aguas que otorga un mayor grado de humedad al subsuelo. Favoreciendo, asimismo, la recarga de los acuíferos subterráneos; muchos de los cuales están amenazados por la salinización o intrusión marina debido a la sobrexplotación a que están sometidos. Numerosos son los proyectos orientados al conocimiento de la singularidad de estos paisajes y las repercusiones desde el punto de vista de la flora y la fauna. El diseño de indicadores para estimar la conservación de suelo y agua por los sistemas rurales tradicionales y las consecuencias derivadas de su abandono es uno de los objetivos del Traditional Knowledge World Bank. Su aplicación a las políticas agrícolas de la Unión Europea, es incipiente.

Las repercusiones de estas iniciativas han sido en muchos casos mediocres y, en otros, incipiente. Su implementación choca con cuestiones como el desconocimiento de la estructuración histórica de estos paisajes, las inversiones costosas en términos económicos e, incluso, la «desaparición de saberes» que dificultaría la realización de algunas prácticas de la ordenación territorial tradicional. Todo ello a pesar de los beneficios que resultarían de su adopción a corto y medio plazo.

\section{BIBLIOGRAFÍA}

ASINS, S. (2007): «Los aterrazamientos mediterráneos, paradigma ambiental-agro-cultural». En: El paisaje mediterráneo. Opciones de multifuncionalidad. Santander, Fundación Santander-Central Hispano, 81-91.

ASINS, S. (2009): El paisaje agrario aterrazado: diálogo entre el hombre y el medio ambiente en Petrer (Alicante). Valencia: PUV. 
BERNABÉ i MESTRE, J.M. (1989): «Obras hidráulicas tradicionales en el regadío de Petrer (Valle del Vinalopó)». En: Los paisajes del agua, Universidad de Valencia- Universidad de Alicante, 85-201.

BLANCHEMANCHE, Ph. (1986): Bâtisseurs de paysages: terrassement, épierrement et petite hydraulique agricoles en Europe XVII-XIX siècles. París, Maison des Sciences de l'Homme.

BOX AMORÓS, M. (1995): «Un aprovechamiento tradicional de agua en el Sureste Ibérico: los aljibes», Investigaciones Geográficas, nº 13, 91-106.

BRAUDEL, F. (2004): La Méditerranée: l'espace et l'histoire. Flammarion.

BRUHNES, Jean (1902): L'irrigation dans la Peninsule Iberique et dans l'Afrique du Nord, Paris.

CALVO GARCÍA TORNEL, F. (1968): «La huerta de Murcia y las avenidas del Guadalentín», Revista Papeles del Departamento de Geografía de la Universidad de Murcia, 1, 111-137.

CAVANILLES, A. J. (1795-1797): Observaciones sobre la Historia Natural, Geografía, Agricultura, Población y Frutos del Reyno de Valencia. Madrid, Imprenta Real.

CHAPMAN, R. (1981): «The evidence of prehistoric water control in south-east of Spain», Journal of Arid Environment, $\mathrm{n}^{\mathrm{o}} 1$.

DEFFONTAINES, P. (1972): El Mediterráneo: la tierra, el mar, los hombres. Barcelona, Juventud.

DESPOIS, J. (1949): «L'utilisation des eaux de crue dans les pays arides de l'Afrique du Nord. Example des plaines du Hodna» en Congreso Internacional de Geografía de Lisboa.

FERRE BUENO, E. (1979): El valle del Almanzora. Almería: Diputación Provincialm, 494 pp.

FORD, R. (1981): Manual para los viajeros por los Reinos de Valencia y Murcia y lectores en casa. Madrid, Ed. Turner.

GIL OLCINA, A. (1974): El campo de Lorca. Estudio de Geografía Agraria. Valencia, Facultad de Filosofía y Letras.

GIL OLCINA, A. (1995): La propiedad de aguas perennes en el sureste ibérico. Alicante, Universidad de Alicante.

GÓMEZ ESPÍN, J.M. (2004): Aprovechamiento integral del agua en la rambla de Nogalte (Puerto Lumbreras, Murcia). Murcia, Universidad de Murcia, 190 pp.

GÓMEZ ESPIN, J.M. y GIL MESEGUER, E. (Coords.) (2006): Modelos de sostenibilidad en el uso del agua en la región de Murcia. Murcia, Universidad de Murcia.

GÓMEZ ESPIN, J.M. y GIL MESEGUER, E. (Coords.) (2010): Paisaje y patrimonio generados por galerías y minados en la Región de Murcia. Murcia, Universidad de Murcia.

GÓMEZ ESPÍN, J.M.; LÓPEZ FERNÁNDEZ, J.A. y MONTANER SALAS, Mª. E. (coords.) (2011): Modernización de regadíos, sostenibilidad social y económica. La singularidad de los regadíos del trasvase Tajo-Segura, Universidad de Murcia, 439

GIL MESEGUER, E. (coord.) (2007): Sistemas locales de recursos propios de agua en la Región de Murcia: Minados y Galerías. Murcia: Universidad de Murcia.

GROVE, A.T. y RACKHAM, O. (2001): The nature of Mediterranean Europe.An Ecological History. New Heaven, Yale University Press. 
HERMOSILLA PLA, J. (dir.) (2006): Las galerías drenantes en el sureste de la península ibérica. Uso tradicional del agua y sostenibilidad en el Mediterráneo español. Madrid, Ministerio de Medio Ambiente.

HERMOSILLA PLA, J. (dir.) (2010): Los regadíos históricos españoles. Paisajes culturales, paisajes sostenibles. Madrid: Ministerio de Medio Ambiente y Medio Rural y Marino, 2010. 608 p.

HERNÁNDEZ HERNÁNDEZ, M. (2010): «Terraced landscapes: new uses and dynamics» en III Anglo-Spanish Rural Geography Conference. Canterbury, AGE y Royal Geographical Society, 81-94.

LANDESCHI, G. B. (1807): Saggi di Agricoltura. Florencia, Giugliemo Prate.

LÓPEZ GÓMEZ, A. (1951): «Riegos y cultivos en la Huerta de Alicante», Estudios Geográficos, $\mathrm{n}^{\circ} 41,701-771$.

LLOBET REBERTER, S. (1958): «Utilización del agua en la región semiárida de HuercalOvera (Almería)», Estudios Geográficos, nº 70, 5-21.

MARCO MOLINA, J. A. y VERA REBOLLO, J. F. (1988): «Impactos de los usos del suelo y erosión en las cuencas vertientes del sur del País Valenciano», Investigaciones Geográficas, $\mathrm{n}^{\mathrm{o}} 6,7-32$.

MARCO MOLINA, J. A. y MORALES GIL, A. (1995): «Terrazas de cultivo abandonadas en el sureste peninsular», Investigaciones Geográficas, n ${ }^{\circ} 13,81-90$.

MARTÍN GALINDO, J. L. (1988): Almería, paisajes agrarios. Espacio y sociedad: de la agricultura morisca a los enarenados e invernaderos actuales. Valladolid, Universidad de Valladolid.

MARTÍNEZ DE AZAGRA, A. (1996): Diseño de sistemas de cosecha de agua para la repoblación forestal. Madrid, Mundi Prensa.

MARTÍNEZ FERNÁNDEZ, J. y ESTEVE SELMA, M.A. (coords.) (2002). Agua, regadío y sostenibilidad en el Sudeste Ibérico. Bilbao: Bakeaz.

MARTÍNEZ FERNÁNDEZ, J. y ESTEVE SELMA, M.A. (2002): Evolución de los regadíos tradicionales del sudeste ibérico: aplicación de un modelo de simulación dinámica. In III Congreso Ibérico sobre gestión y planificación de Aguas. La directiva marco del agua: realidades y futuros. Sevilla,13-17 de noviembre de 2002. Disponible: http://congreso. us.es/ciberico/archivos_html/sevillacomu5.1.htm.

MATA OLMO, R. Agricultura, paisaje y gestión del territorio. Polígonos. Revista de Geografía, 2004, no 14 , p. 97-137.

MATA OLMO, R. y FERNÁNDEZ MUÑOZ, S. (2010): «paisajes y patrimonios culturales del agua. La salvaguarda del valor patrimonial de los regadíos tradicionales», Scripta Nova, vol. XIV, núm. 337. Disponible: http://www.ub.edu/geocrit/sn/sn-337.htm.

MONDEJAR SÁNCHEZ, J. M. (2010): El riego de boqueras: una técnica hidráulica para la gestión ambiental en territorios semiáridos y lucha contra la desertificación. Tesis doctoral (inédita).

MORALES GIL, A. (1969): El riego con aguas de avenida en las laderas subáridas», Papeles del Departamento de Geografía $\mathrm{n}^{\mathrm{o}}$ 1, 167-183.

MORALES GIL, A. y BOX AMORÓS, M. (1986): «El aprovechamiento del agua y los suelos en un dominio semiárido: la cuenca del barranco Blanco. Agost. (Alicante)», Investigaciones geográficas, $\mathrm{n}^{\circ}$ 4, 7-24. 
PAYÁ POVEDA, J.Mª (1990): «Agricultura y propiedad de la tierra en Petrer en 1900», Bitrir $n^{\circ} 13-14,209-233$.

ROMAN DEL CERRO, J.L. (1984): Alicante, 1881-1980. Alicante, Ed. Caja de Ahorros Provincial de Alicante, vol. VI.

SOLER PASCUAL, E. (1994): El País Valenciano a fines del siglo XVIII: Carlos Beramendi y Freyre. Alicante: Instituto de Cultura Juan Gil-Albert.

TORRES FONTES, J. (1960): Repartimiento de la Huerta y Campo de Murcia en el siglo XIII. Murcia, CSIC.

VILA VALENTÍ, J. (1961): «L'irrigation per nappes pluviales dans le Sud-est de l'Espagne», Mediterrannée num 2,19-31. 
